\title{
Review of: "Effects of cannabidiol on activated immune-inflammatory pathways in major depressive patients and healthy controls"
}

\author{
Emanuela Mazzon
}

Potential competing interests: The author(s) declared that no potential competing interests exist.

Rachayon M. et al. evaluated the effects of cannabidiol (CBD) in culture supernatant of stimulated whole blood of depressed patients with activation of the immune-inflammatory response system (IRS) and compensatory immune-regulatory system (CIRS). Specifically, it was examined the anti-inflammatory effects of CBD in IRS and CIRS profile and cytokines, immune-related neurotoxicity, chemokines and growth factor. Overall, this is a good area of research in pharmacology as you demonstrated the complex immunomodulatory effects of CBD.

Please, find below my broad comments on the manuscript:

Overall, the article is well organized and the rationale behind the study is lined out clearly. However, there are some weaknesses, which are pointed out in the following:

Major comments

- The study aims to evaluate the effects of CBD on activated immune-inflammatory pathways in patients with major depression and healthy controls. However, CBD was not administered directly to patients but was tested on whole blood culture supernatant collected from patients. The study aims to evaluate the effects of CBD on activated immune-inflammatory pathways in patients with major depression and healthy controls. However, CBD was not administered directly to patients but was tested on whole blood culture supernatant collected from patients. For this reason, in my opinion, the title is inappropriate it would be appropriate to modify it in such a way as to immediately understand that the effects of CBD have not been tested on a cohort of patients but have been examined whole blood

- In my opinion, in the introduction, the authors should write a general sentence that better highlights the importance of medicinal plants in major depressive disorder and major depressive episodes.

- I suggest the authors add a figure, at end of the discussion, in order to better summarize and clarify the results of your work.

- In my opinion, in the conclusion the transnationality of the study is lacking, so I would better clarify this meaning.

Minor comments:

- In the abstract, the entire name of many abbreviations such as PHA, LPS, IL, G-CSF, INF-, CCL, TNF-, FGF, VEGF are missing. 
- Even in the introduction, the full name of some abbreviations is missing when first written, such as CCL. On the contrary, interleukin-10 could be written directly using the acronym (IL-10) as it was already abbreviated previously. I suggest that you look at the abbreviations throughout the text.

- In the discussion on page 19 , the references $[8,9,50,51]$ could be cited only once at the end of the period "We used a combination of LPS + PHA to stimulate diluted whole blood and analyzed cytokine and growth factor production in culture supernatant. This diluted whole blood method more adequately reflects the in vivo cytokine production than assays on isolated PBMCs. The method used in the present study retains all natural cell-to-cell connections, whereas techniques that isolate PBMC subtypes change the lymphocyte / monocyte ratio thereby affecting cytokine production [8,9,50,51]. 